\title{
A Hybrid-Filter Approach to Turbulence Simulation
}

\author{
Bernie Rajamani • John Kim
}

Received: 17 December 2009 / Accepted: 23 February 2010 / Published online: 20 March 2010

(C) The Author(s) 2010. This article is published with open access at Springerlink.com

\begin{abstract}
Germano (Theor Comput Fluid Dyn 17:225-331, 2004) proposed a hybridfilter approach, which additively combines an LES-like filter operator $(F)$ and a RANS-like statistical operator $(E)$ using a blending function $k: H=k F+(1-k) E$. Using turbulent channel flow as an example, we first conducted a priori tests in order to gain some insights into this hybrid-filter approach, and then performed full simulations to further assess the approach in actual simulations. For a priori tests, two separate simulations, RANS $(E)$ and LES $(F)$, were performed using the same grid in order to construct a hybrid-filtered field $(H)$. It was shown that the extra terms arising out of the hybrid-filtered Navier-Stokes (HFNS) equations provided additional energy transfer from the RANS region to the LES region, thus alleviating the need for the ad hoc forcing term that has been used by some investigators. The complexity of the governing equations necessitated several modifications in order to render it suitable for a full numerical simulation. Despite some issues associated with the numerical implementation, good results were obtained for the mean velocity and skin friction coefficient. The mean velocity profile did not have an overshoot in the logarithmic region for most blending functions, confirming that proper energy transfer from the RANS to the LES region was a key to successful hybrid models. It is shown that Germano's hybrid-filter approach is a viable and mathematically more appealing approach to simulate high Reynolds number turbulent flows.
\end{abstract}

B. Rajamani · J. Kim $(\bowtie)$

Department of Mechanical and Aerospace Engineering, University of California, Los Angeles, Los Angeles, CA 90095-1597, USA

e-mail: jkim@seas.ucla.edu

B. Rajamani

e-mail: bharanidharan.rajamani.2009@anderson.ucla.edu 
Keywords Numerical simulation $\cdot$ Large-eddy simulation $\cdot$ Hybrid approach $\cdot$ Turbulent flow $\cdot$ Channel flow

\section{Introduction}

For a simulation of most turbulent flows of industrial interest, a pure large eddy simulation (LES), let alone direct numerical simulation (DNS), is considered too costly and is estimated to be infeasible until about 2045 (Spalart [15]). The Reynoldsaverage Navier-Stokes (RANS) approach, on the other hand, may not provide the necessary information of interest and sometimes may even be unreliable. Hybrid approaches, which combine the strengths of RANS and LES techniques, have become popular in the last decade. In traditional zonal hybrid methods (e.g., Balaras et al. [3], Wang and Moin [21], Abe [1], Temmerman et al. [19], and Templeton et al. [20]), two separate turbulence models - a RANS model in the near-wall region and a subgrid-scale model for LES in outer regions-are used. The solutions in these two regions are then combined at a suitably chosen near-wall crossover location by matching a secondary quantity such as eddy viscosity. However, it has been widely reported that matching a secondary quantity at the interface is not completely satisfactory; typically, the total stress at the interface is not fully supported by the modeled or resolved stresses. Zonal approaches also lack the ability to properly transfer momentum from the fluctuation-void RANS region to the fluctuationresolved LES region.

Detached eddy simulation (DES) (Spalart et al. [18]; Shur et al. [14]; Nikitin et al. [10]) is different from the zonal approach in that the same eddy viscosity transport equation is used in both RANS and LES regions. The model switches between RANS and LES by virtue of different length scales used for the destruction term in the eddy viscosity transport equation. In the original DES, the switch location was purely determined by the numerical grid used for the simulation. This placed severe constraints on the quality and type of the grid that can be used to obtain reasonable results. This also led to several other problems, most notably, (1) grid-induced separation and (2) non-convergence of solutions upon progressive refinement of the grid. A modified version of DES, known as delayed DES (DDES) (Spalart et al. [17]), was an attempt at addressing these two issues. Nonetheless, the model was still found to be lacking a mechanism for proper momentum and energy transfer between the RANS to the LES regions. A common consequence of this deficiency was an overshoot in the log region of the mean velocity profile of a channel flow simulation, when DES was used as a wall-layer model. Piomelli et al. [11] avoided the log-layer overshoot by adding a stochastic forcing term to the momentum equation. The introduction of the stochastic forcing term improved the momentum transfer significantly and resulted in an induced but sustained production of turbulence fluctuations at the interface. Higher production at the interface increased resolved stresses, resulting in the reduction of velocity gradient there. In the original DES (without stochastic forcing), this velocity gradient was artificially high to compensate for the reduction in resolved stress caused by the lack of fluctuating quantities at the interface. The stochastic forcing, however, was rather arbitrary in that the strength and frequency of forcing required may depend on the grid, flows, and Reynolds numbers. 
The key point in the above discussion is the requirement of additional turbulence fluctuations to sustain the correct level of total turbulent stresses at the interface. We will elaborate on this point later in our discussion. It is Germano's contention [4] that a mathematically correct way of blending two disparate models is to formally develop the properties of a hybrid filter $H$. Such a hybrid filter model is quite different from its parent models. For example, Reynolds shear stress derived as a second moment of velocity fluctuations has a new additional term, and the continuity equation is no longer divergence free. The objective of the present work was to analyze Germano's hybrid-filter approach through a priori tests in order to gain some insights into the model, and then to perform full simulations to further assess the validity of Germano's approach. Our study was also aimed at improving the understanding of the momentum and energy transfer at the interface of hybrid approaches. We provide some analysis of this by examining the production of $H$-field residual kinetic energy near the interface.

This paper is organized as follows: the hybrid-filtered Navier-Stokes equations (HFNS) are first derived in Section 2, results from a priori tests are given in Section 3, full simulation results are presented in Section 4, and a summary and concluding remarks are given in Section 5.

We shall use $(x, y, z)$ or $\left(x_{1}, x_{2}, x_{3}\right)$ to denote streamwise, wall-normal and spanwise directions respectively and $(u, v, w)$ or $\left(u_{1}, u_{2}, u_{3}\right)$ for corresponding velocity components. All variables are non-dimensionalized by the centerline velocity, $U$, and the channel half width unless stated otherwise. The channel extends from -1 to +1 in the wall-normal direction, but for presentation of results we sometimes modify the coordinates so that it extends from 0 to 2 . The superscript "+" denotes a quantity normalized by the viscosity and wall-shear velocity.

\section{Governing Equations}

We reproduce the governing equations from the original paper for completeness. For the hybrid filter, $H=k F+(1-k) E$, if we invoke the assumption of commutativity of filter operator $F$ and the derivative operator $\mathcal{D}_{i}=\frac{\partial}{\partial x_{i}}$ and the fact that $E F=E$, it can be shown that

$$
H \mathcal{D}_{i}=\mathcal{D}_{i} H-\frac{\partial k}{\partial x_{i}}(F-E) .
$$

Applied to the continuity equation, (1) leads to the hybrid-filtered continuity equation (HFCE)

$$
\frac{\partial\left\langle u_{j}\right\rangle_{H}}{\partial x_{j}}=\frac{\partial k}{\partial x_{j}}\left(\left\langle u_{j}\right\rangle_{F}-\left\langle u_{j}\right\rangle_{E}\right),
$$

where the quantity within $\langle>$ represents a hybrid-filtered, LES-filtered or RANSaveraged variable, depending on whether the subscript is $H, F$, or $E$ respectively. Note that the hybrid-filtered field is non-solenoidal. As can be seen in HFCE, there is a need to evaluate the $F$ - and $E$-field quantities from the $H$-field. Since $E$ is the statistical operator, it satisfies the property, $E H=E$. Thus for any scalar or vector $\phi_{i}$

$$
\left\langle\phi_{i}\right\rangle_{E}=\left\langle\left\langle\phi_{i}\right\rangle_{H}\right\rangle_{E} .
$$


The $F$-filtered field can be reconstructed from the definition of $H$-filter as

$$
\left\langle\phi_{i}\right\rangle_{F}=\frac{\left\langle\phi_{i}\right\rangle_{H}-\left\langle\phi_{i}\right\rangle_{E}}{k}+\left\langle\phi_{i}\right\rangle_{E},
$$

or in its other more useful form:

$$
\left\langle\phi_{i}\right\rangle_{F}=\frac{1-k}{k}\left(\left\langle\phi_{i}\right\rangle_{H}-\left\langle\phi_{i}\right\rangle_{E}\right)+\left\langle\phi_{i}\right\rangle_{H} .
$$

When the hybrid filter is applied to the Navier-Stokes equations, we obtain the hybrid-filtered Navier-Stokes (HFNS) equation, which can be written in nondimensional form as

$$
\begin{aligned}
& \frac{\partial\left\langle u_{i}\right\rangle_{H}}{\partial t}+\frac{\partial\left\langle u_{i}\right\rangle_{H}\left\langle u_{j}\right\rangle_{H}}{\partial x_{j}}+\frac{\partial\langle p\rangle_{H}}{\partial x_{i}}-\frac{1}{R e} \frac{\partial^{2}\left\langle u_{i}\right\rangle_{H}}{\partial x_{j}^{2}} \\
& =-\frac{\partial \tau_{H}\left(u_{i}, u_{j}\right)}{\partial x_{j}}+\frac{\partial k}{\partial x_{i}}\left(\langle p\rangle_{F}-\langle p\rangle_{E}\right) \\
& \quad+\frac{\partial k}{\partial x_{j}}\left(\left\langle u_{i}\right\rangle_{F}\left\langle u_{j}\right\rangle_{F}-\left\langle u_{i}\right\rangle_{E}\left\langle u_{j}\right\rangle_{E}\right)+\frac{\partial k}{\partial x_{j}}\left(\tau_{F}\left(u_{i}, u_{j}\right)-\tau_{E}\left(u_{i}, u_{j}\right)\right) \\
& \quad-\frac{2}{\operatorname{Re}} \frac{\partial k}{\partial x_{j}} \frac{\partial}{\partial x_{j}}\left(\left\langle u_{i}\right\rangle_{F}-\left\langle u_{i}\right\rangle_{E}\right)-\frac{1}{\operatorname{Re}} \frac{\partial^{2} k}{\partial x_{j}^{2}}\left(\left\langle u_{i}\right\rangle_{F}-\left\langle u_{i}\right\rangle_{E}\right) .
\end{aligned}
$$

Equation (6) uses the following definition for the nonlinear terms for the $H$ quantities. Written in general form this is

$$
\left\langle u_{i} u_{j}\right\rangle_{\mathcal{O}}=\left\langle u_{i}\right\rangle_{\mathcal{O}}\left\langle u_{j}\right\rangle_{\mathcal{O}}+\tau_{\mathcal{O}}\left(u_{i}, u_{j}\right)
$$

in which $\mathcal{O}$ could be one of $H, F$ or $E$, and $\tau_{H}$ and $\tau_{F}$ are subgrid scale stresses at the $H$ and $F$ levels, while $\tau_{E}$ is the Reynolds stress tensor at the $E$ level. The $H$-field SGS stress, $\tau_{H}$ can be derived as

$$
\tau_{H}\left(u_{i}, u_{j}\right)=k \tau_{F}\left(u_{i}, u_{j}\right)+(1-k) \tau_{E}\left(u_{i}, u_{j}\right)+G_{i j}
$$

where

$$
G_{i j}=k(1-k)\left(\left\langle u_{i}\right\rangle_{F}-\left\langle u_{i}\right\rangle_{E}\right)\left(\left\langle u_{j}\right\rangle_{F}-\left\langle u_{j}\right\rangle_{E}\right) .
$$

We shall discuss more about this term later. Finally, the anisotropic part of $\tau_{F}$ and $\tau_{E}$ are given using the corresponding strain rate and eddy viscosity:

$$
\begin{gathered}
\tau_{F}^{a}=-2 v_{F}\left\langle S_{i j}\right\rangle_{F} \\
\tau_{E}^{a}=-2 v_{E}\left\langle S_{i j}\right\rangle_{E} .
\end{gathered}
$$

The crossover occurs according to the specified blending function $k$. Reconstruction of $E$-field from $H$-field is a statistical averaging procedure, while the $F$-field is reconstructed according to (4). More discussions on the reconstruction and the choice of a blending function will be presented in Section 4. 
Applying the above definitions to (6) we get

$$
\begin{gathered}
\frac{\partial\left\langle u_{i}\right\rangle_{H}}{\partial t}+\frac{\partial\left\langle u_{i}\right\rangle_{H}\left\langle u_{j}\right\rangle_{H}}{\partial x_{j}}+\frac{\widehat{\partial\langle P\rangle_{H}}}{\partial x_{i}}-\frac{\partial}{\partial x_{j}}\left[\frac{1}{R e} \frac{\partial\left\langle u_{i}\right\rangle_{H}}{\partial x_{j}}-\tau_{H}^{a}\left(u_{i}, u_{j}\right)\right] \\
=+\frac{\partial k}{\partial x_{j}}\left(\left\langle u_{i}\right\rangle_{F}\left\langle u_{j}\right\rangle_{F}-\left\langle u_{i}\right\rangle_{E}\left\langle u_{j}\right\rangle_{E}\right)+\frac{\partial k}{\partial x_{j}}\left(\tau_{F}^{a}\left(u_{i}, u_{j}\right)-\tau_{E}^{a}\left(u_{i}, u_{j}\right)\right) \\
-\frac{2}{\operatorname{Re}} \frac{\partial k}{\partial x_{j}} \frac{\partial}{\partial x_{j}}\left(\left\langle u_{i}\right\rangle_{F}-\left\langle u_{i}\right\rangle_{E}\right)-\frac{1}{\operatorname{Re}} \frac{\partial^{2} k}{\partial x_{j}^{2}}\left(\left\langle u_{i}\right\rangle_{F}-\left\langle u_{i}\right\rangle_{E}\right),
\end{gathered}
$$

where

$$
\widetilde{\frac{\partial\langle P\rangle_{H}}{\partial x_{i}}}=\frac{\partial\langle P\rangle_{H}}{\partial x_{i}}-\frac{\partial k}{\partial x_{i}}\left(\langle P\rangle_{F}-\langle P\rangle_{E}\right),
$$

and

$$
\langle P\rangle_{\mathcal{O}}=\langle p\rangle_{\mathcal{O}}+\frac{2}{3} k_{\mathcal{O}}
$$

with $\mathcal{O}$ representing one of $H, F$ or $E$-filtered quantities, and $k$ representing the trace of the respective Reynolds stress tensor depending on whether the suffix is $H, F$ or $E$. Equation (12) is different from that in Germano [4] in that all terms involving pressure (including the isotropic part of the subgrid stresses as is commonly done) are combined into one term. Written this way, $\frac{\partial\langle P\rangle_{H}}{\partial x_{i}}$, which constrains the $H$-field velocity to satisfy the HFCE, can be solved in the same manner as pressure in LES (i.e., via a Poisson equation).

\section{A Priori Tests}

In order to gain some insights into the hybrid-filter approach, we first conducted somewhat unique a priori tests. We performed two separate simulations, RANS and LES, of a channel flow at $R e_{\tau}=560$ using the same $129 \times 129 \times 129$ grid. For the RANS simulation, we used the Spalart-Allmaras model [16], while the dynamic Smagorinsky model [5] was used for the LES. These two solutions were combined using the blending function $k$ to obtain the $H$-field. The blending function was quite similar to the $f_{d}$ function of Spalart in DDES [17],

$$
k=(1.0+\epsilon)-\tanh \left(8 r_{d}^{3}+\epsilon\right),
$$

where $r_{d}$ is the same function used in DDES. Here $\epsilon$ could be a small positive number to allow $k$ to start from a non-zero value. For the results presented, we used a $k$ with $\epsilon=0.15$ or with $\epsilon=0$. These two blending functions are shown in Fig. 1.

\subsection{The continuity equation for hybrid velocity field}

The HFCE (2) is interesting because it is not divergence free. This is owing to the non-commutativity of the $H$-filter and derivative operators as seen from (1). At 
Fig. 1 Blending functions: solid line $k=0$ near the wall; dotted line $k=0.15$ near the wall

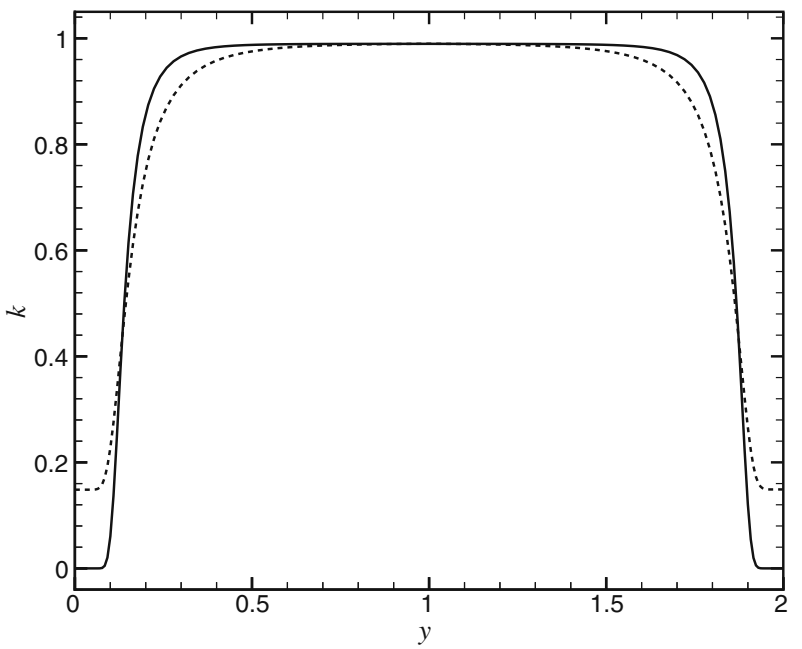

first glance, it is difficult to interpret the meaning of a non-zero divergence in the continuity equation because a control volume analysis of an arbitrary section of the channel fails to conserve mass. To understand this we first note that

$$
\begin{aligned}
\left\langle\frac{\partial\left\langle u_{j}\right\rangle_{H}}{\partial x_{j}}\right\rangle_{E} & =\left\langle\frac{\partial k}{\partial x_{j}}\left(\left\langle u_{j}\right\rangle_{F}-\left\langle u_{j}\right\rangle_{E}\right)\right\rangle_{E} \\
\frac{\partial\left\langle\left\langle u_{j}\right\rangle_{H}\right\rangle_{E}}{\partial x_{j}} & =\frac{\partial k}{\partial x_{j}}\left(\left\langle\left\langle u_{j}\right\rangle_{F}\right\rangle_{E}-\left\langle\left\langle u_{j}\right\rangle_{E}\right\rangle_{E}\right) \\
\frac{\partial\left\langle u_{j}\right\rangle_{E}}{\partial x_{j}} & =0
\end{aligned}
$$

and hence HFCE is divergence free on the average. This can also be seen in Fig. 2, which shows the right-hand side of the spatially-averaged continuity equation as a function of time at different wall-normal locations. At all wall-normal locations the time average tends to be zero.

Strictly speaking, this non-solenoidal continuity equation applies to all hybrid models, although, to the best of our knowledge, it is usually ignored. Consider a case where a RANS model is used in the near wall region, and an LES model is used in the outer region, and the two regions are blended smoothly using a blending function $k$. In such a case, the velocity profile is defined as,

$$
\tilde{u}_{i}=\left\{\begin{array}{lc}
\left\langle u_{i}\right\rangle & \text { RANS } \\
k \bar{u}_{i}+(1-k)\left\langle u_{i}\right\rangle & \text { interface } \\
\bar{u}_{i} & \text { LES }
\end{array}\right.
$$


Fig. $2 E$-averaged continuity equation plotted for different wall-normal locations

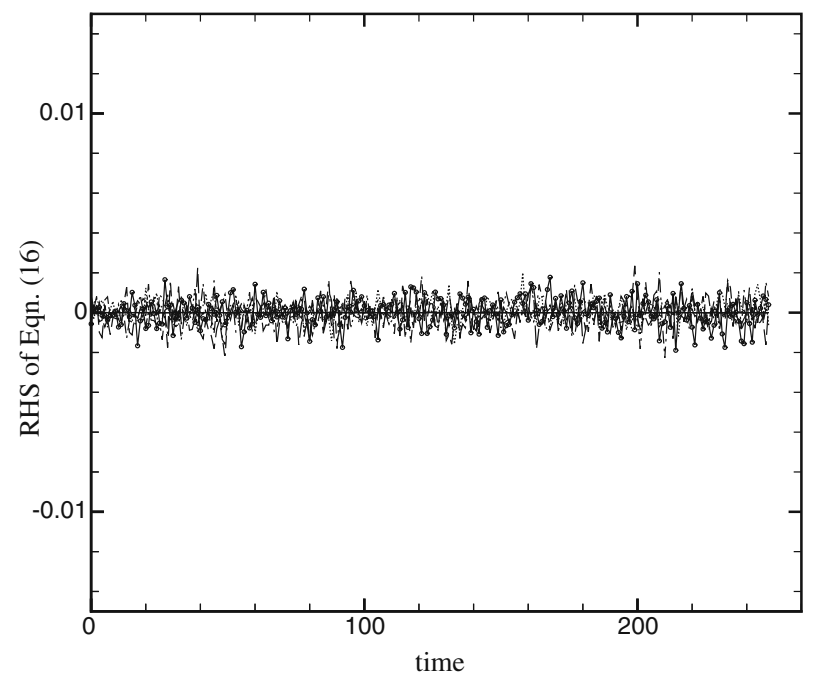

where \langle\rangle denotes a RANS-averaged field and "bar" represents an LES-filtered field. The continuity equation for the velocity field, $\tilde{u}_{i}$, in this case yields

$$
\frac{\partial \tilde{u}_{i}}{\partial x_{i}}=\left\{\begin{array}{lc}
\frac{\partial\left\langle u_{i}\right\rangle}{\partial x_{i}}=0 & \text { RANS; } \\
\frac{\partial k}{\partial x_{i}}\left(\bar{u}_{i}-\left\langle u_{i}\right\rangle\right) \neq 0 & \text { interface; } \\
\frac{\partial \bar{u}_{i}}{\partial x_{i}}=0 & \text { LES. }
\end{array}\right.
$$

While equating the divergence to zero is acceptable in the RANS and LES regions, a divergence-free condition amounts to setting both the RANS and LES velocities equal in the interface region. Obviously, this is inaccurate. The current form of the continuity equation is more accurate in situations where a separate RANS and LES models are combined using a smooth blending function.

\subsection{Hybrid stresses}

The HFNS contains the $H$-field subgrid-scale stress term (8), which consists of three terms. The first two terms of these three are what is expected of a direct blending of RANS and LES. The third term, $G_{i j}$ which will be referred to as Germano stresses hereinafter, can be quite significant at the interface owing to the non-zero fluctuation-like quantity, $\left(\left\langle u_{i}\right\rangle_{F}-\left\langle u_{i}\right\rangle_{E}\right)$. To examine the contribution of this term we considered the anisotropic part of $\tau_{H}$, which is given as

$$
\tau_{H}^{a}=-2 k v_{F}\left\langle S_{i j}\right\rangle_{F}-2(1-k) v_{E}\left\langle S_{i j}\right\rangle_{E}+k(1-k) G_{i j}^{a},
$$

where $G_{i j}$ is given by (9). For the a priori test, we obtained the strain rates, $v_{F}, v_{E}$ and the exact value of $G_{i j}^{a}$ term directly from the LES and RANS solutions. Thus we could calculate $\tau_{H}^{a}$ exactly. Figure 3 a shows the modeled Reynolds shear stress, while Fig. $3 \mathrm{~b}$ shows the total (modeled + resolved) stress, both with and without the $G_{i j}^{a}$ 

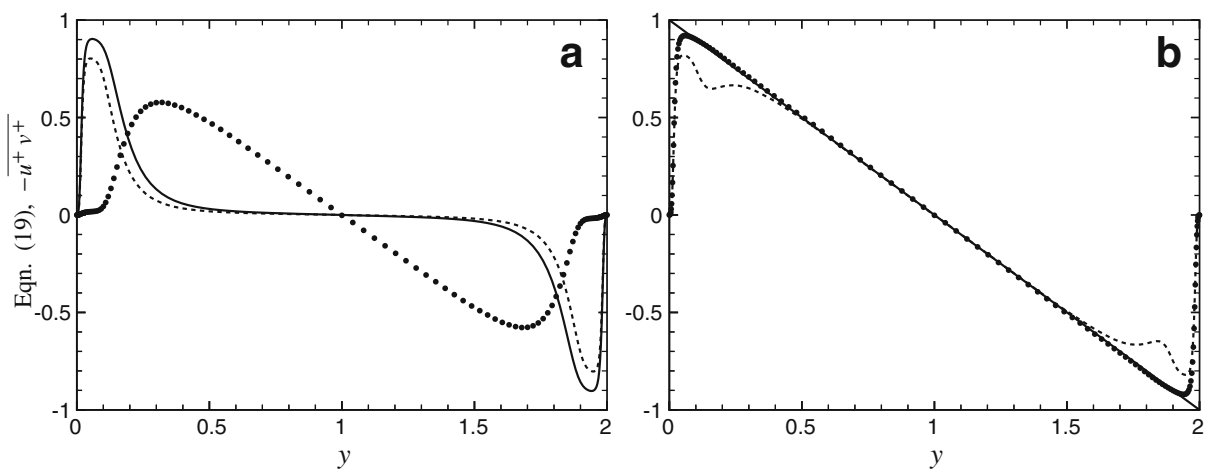

Fig. 3 Reynolds shear stresses. a Solid line modeled stress with the $G_{i j}^{a}$ term ; dotted line modeled stress without the $G_{i j}^{a}$ term; circle resolved stress. b Circle total Reynolds shear stress with $G_{i j}^{a}$ term; dotted line total stress without the $G_{i j}^{a}$ term; solid line (1-y)

term. It is seen that the modeled stress without the Germano stress term is smaller than that with it, and the difference is more pronounced when $k$ is neither zero nor 1 (see Fig. 1). This trend is more clearly shown in Fig. 3b, wherein the total stress without the $G_{i j}^{a}$ term is seen to have a defect resulting in an oddly-shaped curve. This test reveals that the Germano stress term plays a critical role in its contribution to the total shear stress. Ignoring this term and blending any two models directly may lead to significant discrepancy in the total Reynolds shear stress. On the other hand, for a DES-like wall layer model, wherein a single eddy viscosity transport equation is used, such a trend is not explicitly revealed. Nevertheless, the problem owing to the lack of this term exists in other forms: depletion of modeled stress for ambiguous grid densities, improper transport of wall-normal momentum fluxes, poor skin-friction coefficient, and overly large velocity gradients at the interface leading to an overshoot in the log-law region.

\subsection{Contributions of extra terms in HFNS}

In this section we analyze the contribution of the extra terms, i.e., the terms that contain the derivatives of $k$ in the HFNS equations. For this let us define

$$
\begin{aligned}
\mathcal{Q}_{i}= & \frac{\partial k}{\partial x_{j}}\left(\left\langle u_{i}\right\rangle_{F}\left\langle u_{j}\right\rangle_{F}-\left\langle u_{i}\right\rangle_{E}\left\langle u_{j}\right\rangle_{E}+\tau_{F}^{a}-\tau_{E}^{a}-\frac{2}{R e} \frac{\partial}{\partial x_{j}}\left(\left\langle u_{i}\right\rangle_{F}-\left\langle u_{i}\right\rangle_{E}\right)\right) \\
& -\frac{1}{R e} \frac{\partial^{2} k}{\partial x_{j}^{2}}\left(\left\langle u_{i}\right\rangle_{F}-\left\langle u_{i}\right\rangle_{E}\right)
\end{aligned}
$$

and then the right hand side of the HFNS is

$$
\mathcal{R}_{i}=-\frac{\partial \tau_{H}^{a}}{\partial x_{j}}+\mathcal{Q}_{i} .
$$


Now the ratio $\frac{\mathcal{Q}_{i}}{\mathcal{R}_{i}}$ will represent the fractional contribution due to the extra terms.

Figure 4 shows the instantaneous values of the ratio $\frac{\mathcal{Q}_{i}}{\mathcal{R}_{i}}$ at different wall-normal locations. Note that for the blending function chosen, the extra terms contribute only near the RANS-LES interface. At $y^{+}=45$, where the blending function just starts to increase from zero, the contribution of the extra terms is about $7 \%$. At $y^{+} \approx 90$, where about $55 \%$ of $H$-filtered velocity is due to LES, the extra terms contribute about $33 \%$. In the next two locations, $y^{+} \approx 110$ and $y^{+} \approx 160$, where LES is about $79 \%$ and $92 \%$ respectively, the contribution due to the extra terms is reduced to $15 \%$ and $8 \%$. Recognizing that $\mathcal{Q}_{i}$ is significant near the RANS/LES region only, we conclude that $\mathcal{Q}_{i}$ plays a similar role to the ad hoc forcing term added into traditional hybrid approaches. However, $\mathcal{Q}_{i}$ in the hybrid-filter approach arises naturally as a consequence of formal blending of two filtered fields.

\subsection{Instantaneous velocity field}

The transport of turbulence fluctuations in the wall-normal direction is a major problem in hybrid models. This is understandable because the fluctuation-void RANS simulation and fluctuation-resolved LES simulation are merged at the interface "forcibly" in usual hybrid models. The only way the fluctuations can be transported has been by artificially including additional fluctuations at the interface. This was done in Piomelli et al. [11]. In the $H$-filter approach, this is accomplished by three means: the smooth blending function, the Germano stresses, and the extra terms in the momentum equation. Notably, all three terms are most active only near the interface. The $H$-filter adjusts the amount of fluctuations transferred from the fully

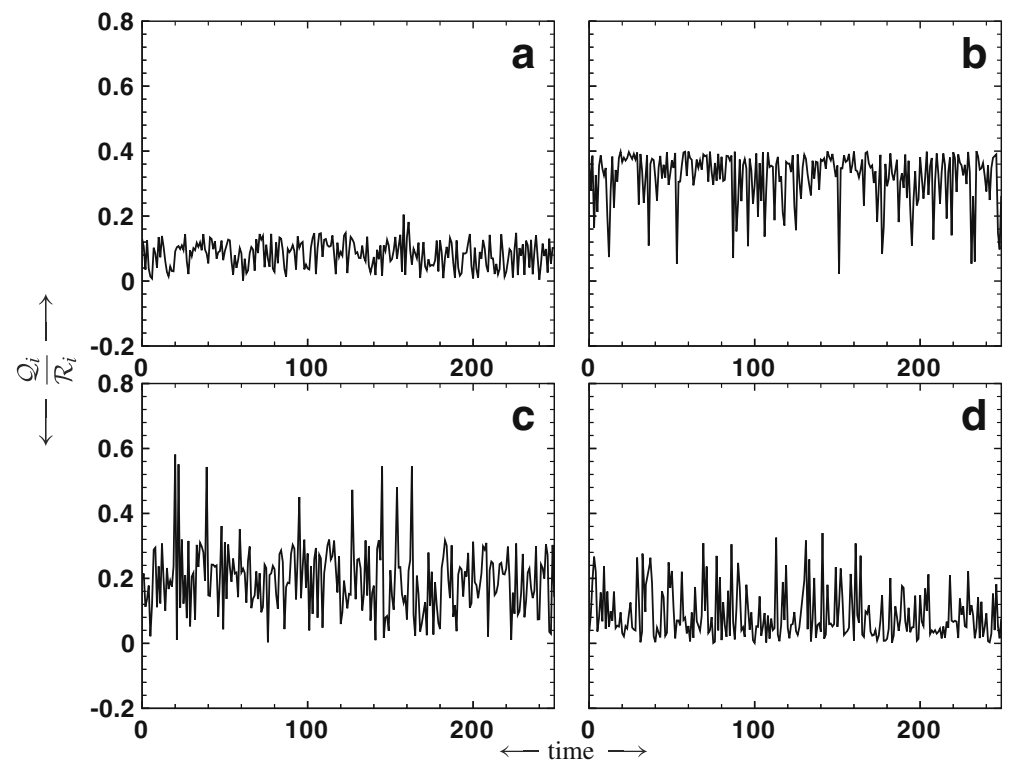

Fig. 4 Contributions of extra terms to the HFNS equation at different wall-normal locations: a $y^{+}=$ $45 ; \mathbf{b} y^{+}=90 ; \mathbf{c} y^{+}=110 ; \mathbf{d} y^{+}=160$ 
RANS region to the fully LES region, depending on the local grid density and the blending function used. In our past work, and as reported by Piomelli [11], we observed artificially long streamwise streaks in velocity fluctuations in the nearwall region for DES. These were blamed for adversely affecting the momentum and energy transport at the interface, causing large velocity gradients and resulting in an overshoot in the log-layer. In the current $H$-filter approach, this deficiency is expected to be diminished, if not completely eliminated, and thereby the long streamwise streaks would break down, yielding results similar to those obtained by stochastic forcing case of Piomelli [11]. In order to examine this phenomenon, we constructed the $H$-field fluctuation defined as,

$$
\left\langle u_{i}^{\prime}\right\rangle_{H}=\left\langle u_{i}\right\rangle_{H}-\left\langle\left\langle u_{i}\right\rangle_{H}\right\rangle_{E}
$$

Contours of this quantity are shown in Fig. 5. At $y^{+}=6$, very close to the wall, we see that the long streamwise streaks are broken down as was seen in Piomelli et al.'s stochastic forcing case, thus confirming the sufficiency of "forcing" at the interface in the $H$-filter approach. At other locations, LES has dominant contributions to the total solution and contours show turbulent fluctuations similar to those observed in LES.

\section{5 $\mathrm{H}$-filtered turbulent kinetic energy budget}

The H-filtered energy equation is obtained by multiplying (12) by $\left\langle u_{i}\right\rangle_{H}$ :

$$
\frac{D_{H}\langle E\rangle_{H}}{D t}+\left\langle u_{j}\right\rangle_{H} \frac{\widetilde{\partial P\rangle_{H}}}{\partial x_{j}}+\frac{\partial\left\langle T_{j}\right\rangle_{H}}{\partial x_{j}}=\langle\varepsilon\rangle_{H}+\langle\mathcal{P}\rangle_{H}+\langle Q\rangle_{H},
$$

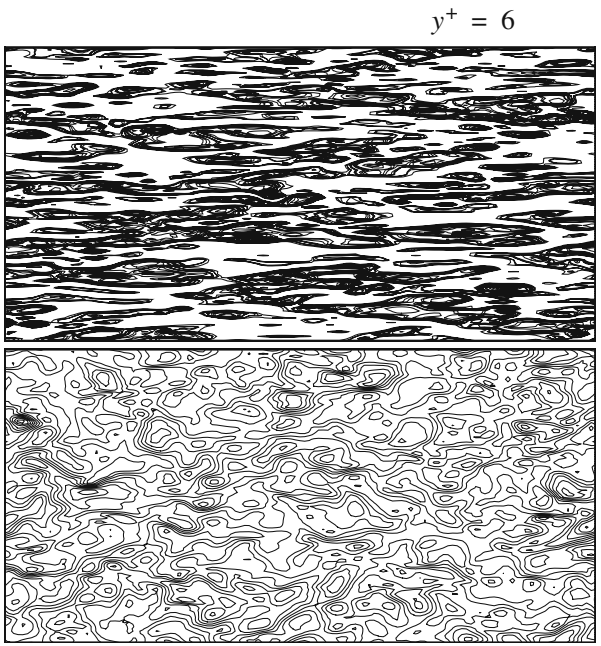

$$
y^{+}=220
$$

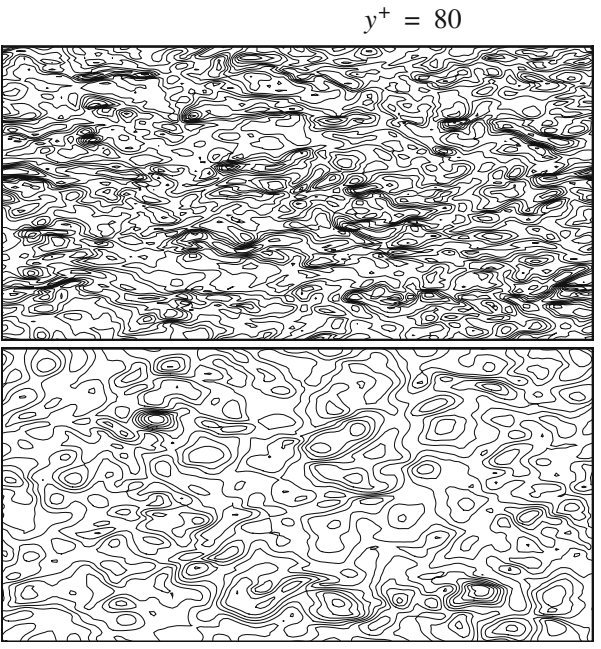

$$
y^{+}=500
$$

Fig. 5 Contours of streamwise velocity fluctuations, $R e_{\tau}=560$. Plotted at four different wall-normal locations as shown 
where

$$
\begin{gathered}
\frac{D_{H}}{D t}=\frac{\partial}{\partial t}+\left\langle u_{j}\right\rangle_{H} \frac{\partial}{\partial x_{j}}, \quad\langle E\rangle_{H}=\frac{\left\langle u_{j}\right\rangle_{H}\left\langle u_{j}\right\rangle_{H}}{2}, \quad\langle\varepsilon\rangle_{H}=-\frac{2}{R e}\left\langle S_{i j}\right\rangle_{H}\left\langle S_{i j}\right\rangle_{H}, \\
\left\langle T_{j}\right\rangle_{H}=\left\langle u_{i}\right\rangle_{H} \tau_{H}^{a}\left(u_{i}, u_{j}\right)-\frac{2}{R e}\left\langle u_{i}\right\rangle_{H}\left\langle S_{i j}\right\rangle_{H}, \quad\langle\mathcal{P}\rangle_{H}=\tau_{H}^{a}\left(u_{i}, u_{j}\right)\left\langle S_{i j}\right\rangle_{H}, \\
\langle Q\rangle_{H}=\frac{\partial k}{\partial x_{j}}\left[\left\langle u_{i}\right\rangle_{H}\left(\left\langle u_{i} u_{j}\right\rangle_{F}-\left\langle u_{i} u_{j}\right\rangle_{E}\right)-2\langle E\rangle_{H}\left(\left\langle u_{j}\right\rangle_{F}-\left\langle u_{j}\right\rangle_{E}\right)-\frac{2}{R e}\left\langle u_{i}\right\rangle_{H} \frac{\partial\left\langle u_{i}\right\rangle_{F}}{\partial x_{j}}\right]- \\
-\frac{1}{\operatorname{Re}} \frac{\partial^{2} k}{\partial x_{j}^{2}}\left\langle u_{i}\right\rangle_{H}\left(\left\langle u_{i}\right\rangle_{F}-\left\langle u_{i}\right\rangle_{E}\right)-\frac{1}{\operatorname{Re}}\left\langle u_{i}\right\rangle_{H} \frac{\partial}{\partial x_{i}}\left(\frac{\partial k}{\partial x_{j}}\left(\left\langle u_{j}\right\rangle_{F}-\left\langle u_{j}\right\rangle_{E}\right)\right) .
\end{gathered}
$$

The term $\langle Q\rangle_{H}$ arises owing to the extra terms in the momentum equation and owing to the non-divergence-free form of the continuity equation. The corresponding LES equation for the filtered velocity field is

$$
\frac{\bar{D} E_{f}}{D t}+\bar{u}_{j} \frac{\partial \bar{p}}{\partial x_{j}}+\frac{\partial \mathcal{T}_{j}}{\partial x_{j}}=\varepsilon_{f}+\mathcal{P},
$$

where

$$
\begin{aligned}
\frac{\bar{D}}{D t} & =\frac{\partial}{\partial t}+\bar{u}_{j} \frac{\partial}{\partial x_{j}}, \\
E_{f} & =\frac{\bar{u}_{j} \bar{u}_{j}}{2}, \\
\mathcal{T}_{j} & =\bar{u}_{i} \tau_{i j}^{a}-\frac{2}{R e} \bar{u}_{i} \bar{S}_{i j}, \\
\varepsilon_{f} & =-\frac{2}{R e} \bar{S}_{i j} \bar{S}_{i j}, \\
\mathcal{P} & =\tau_{i j}^{a} \bar{S}_{i j} .
\end{aligned}
$$

Like the momentum equations, the kinetic energy equation of the $H$-filtered (23) and LES (24) fields have analogous terms. The only difference is the appearance of the term $\langle Q\rangle_{H}$ in the former. As in the momentum equation, these extra terms are effective only in the interface region, and therefore adds or removes the energy only in this region. Whereas the net contribution of the extra terms in the momentum equation is zero in the mean, the term $\left\langle\langle Q\rangle_{H}\right\rangle_{E}$ in the energy equation is not. In this sense, this model is quite similar to Piomelli et al.'s [11] stochastic backscatter model, which adds significant energy (without adding net mean momentum) at the interface. Figure 6 shows the mean $\langle Q\rangle_{H}$, which is non-zero only at the interface. It is worth noting that this extra term removes energy from the region facing the RANS region and adds energy to the region facing the LES region, thus providing wall-normal energy transport that is absent in conventional hybrid approaches.

Next, we examine the terms $\mathcal{P}=\tau_{i j}^{a} \bar{S}_{i j}$ (bar represents LES filtering) and $\langle\mathcal{P}\rangle_{H}=$ $\tau_{H}^{a}\left(u_{i}, u_{j}\right)\left\langle S_{i j}\right\rangle_{H}$. The $\langle\mathcal{P}\rangle_{H}$ term, which is actually a sink in the $H$-filtered kinetic energy equation (even though it appears with a positive sign), will be a source in the residual kinetic energy equation. In other words, it represents the energy transfer 
Fig. 6 Solid line production of residual kinetic energy for LES; solid-filled circle-solid line production of the residual kinetic energy for $H$-field; solid-open circle-solid line, $\langle Q\rangle_{H}$ in (23)

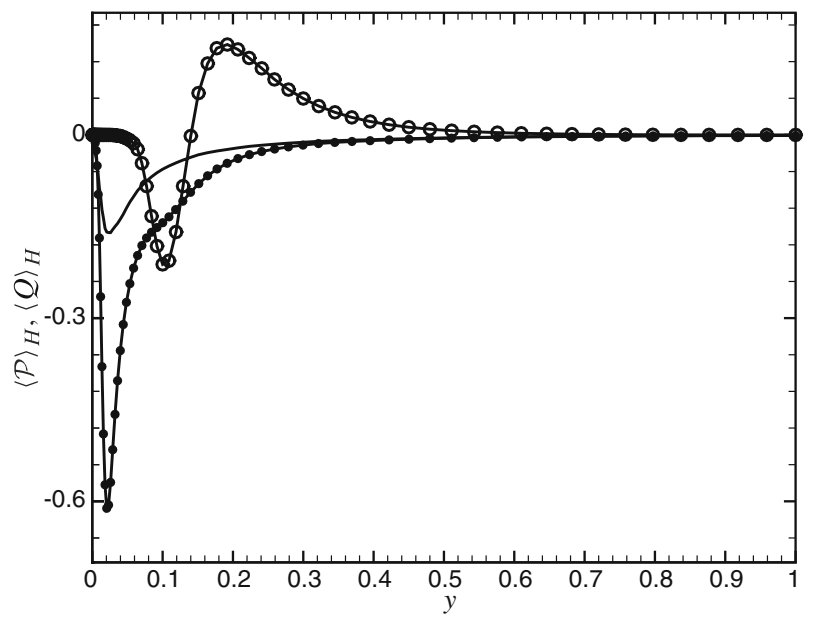

from resolvable-scale motions to subgrid-scale motions. Mean values of $\mathcal{P}$ and $\langle\mathcal{P}\rangle_{H}$ are shown in Fig. 6. Note that the magnitude of $\langle\mathcal{P}\rangle_{H}$ is much larger than that of $\mathcal{P}$. This is because the former depends on RANS and LES eddy viscosities while the latter depends on LES eddy viscosity only. Recall that $\langle\mathcal{P}\rangle_{H}$ represents the energy transfer from $H$-field to subgrid field that includes RANS and LES subgrid scales, whereas $\mathcal{P}$ represents the energy transfer from LES resolved scales to subgrid scales.

\section{Simulations}

The ultimate success (or failure) of a numerical procedure and turbulence model can be measured by its performance in actual simulations. In this section, we present how the hybrid-filter approach was implemented, along with some issues that arose during the implementation, and then we discuss simulation results.

\subsection{Hybrid-filtered Navier-Stokes equations}

Starting from (12), the governing equations can be written as

$$
\begin{gathered}
\frac{\partial\left\langle u_{i}\right\rangle_{H}}{\partial t}+\frac{\partial\left\langle u_{i}\right\rangle_{H}\left\langle u_{j}\right\rangle_{H}}{\partial x_{j}}+\frac{\widetilde{\mathrm{d}\langle P\rangle_{E}}}{\mathrm{~d} x_{1}} \delta_{1 i}+\frac{\widehat{\partial\left\langle P^{\prime}\right\rangle_{H}}}{\partial x_{i}}-\frac{\partial}{\partial x_{j}}\left[\frac{1}{R e} \frac{\partial\left\langle u_{i}\right\rangle_{H}}{\partial x_{j}}-\tau_{H}^{a}\left(u_{i}, u_{j}\right)\right] \\
=+\frac{\partial k}{\partial x_{j}}\left(\left\langle u_{i}\right\rangle_{F}\left\langle u_{j}\right\rangle_{F}-\left\langle u_{i}\right\rangle_{E}\left\langle u_{j}\right\rangle_{E}\right)+\frac{\partial k}{\partial x_{j}}\left(\tau_{F}^{a}\left(u_{i}, u_{j}\right)-\tau_{E}^{a}\left(u_{i}, u_{j}\right)\right) \\
\quad-\frac{2}{R e} \frac{\partial k}{\partial x_{j}} \frac{\partial}{\partial x_{j}}\left(\left\langle u_{i}\right\rangle_{F}-\left\langle u_{i}\right\rangle_{E}\right)-\frac{1}{\operatorname{Re}} \frac{\partial^{2} k}{\partial x_{j} \partial x_{j}}\left(\left\langle u_{i}\right\rangle_{F}-\left\langle u_{i}\right\rangle_{E}\right)
\end{gathered}
$$

where the pressure gradient term is split into its mean and fluctuation.

The subgrid-scale stresses at the $F$ and $E$ levels in (26) are modeled, respectively, through the dynamic Smagrorinsky model and Spalart-Allmaras eddy viscosity 
model. The subgrid-scale stress at the $H$ level could be computed directly from

$$
\tau_{H}^{a}=k \tau_{F}^{a}+(1-k) \tau_{E}^{a}+k(1-k) G_{i j}^{a} .
$$

It turned out, however, that the $H$-level eddy viscosity determined this way was not dissipative enough, resulting in noisy or unstable solutions. Instead, we used an equivalent eddy viscosity model for $H$-level subgrid-scale stress,

$$
\tau_{H}^{a}=-2 v_{H}\left\langle S_{i j}\right\rangle_{H} .
$$

Substituting (28) and the corresponding eddy viscosity model for $F$ and $E$ levels into (27) yields,

$$
-2 v_{H}\left\langle S_{i j}\right\rangle_{H}=-2 k v_{F}\left\langle S_{i j}\right\rangle_{F}-2(1-k) v_{E}\left\langle S_{i j}\right\rangle_{E}+k(1-k) G_{i j}^{a} .
$$

By contracting the above equation with $\left\langle S_{i j}\right\rangle_{H}$

$$
\begin{aligned}
-2 v_{H}\left\langle S_{i j}\right\rangle_{H}\left\langle S_{i j}\right\rangle_{H}= & -2 k v_{F}\left\langle S_{i j}\right\rangle_{F}\left\langle S_{i j}\right\rangle_{H}-2(1-k) v_{E}\left\langle S_{i j}\right\rangle_{E}\left\langle S_{i j}\right\rangle_{H} \\
& +k(1-k) G_{i j}^{a}\left\langle S_{i j}\right\rangle_{H},
\end{aligned}
$$

we get

$$
v_{H}=\frac{k v_{F}\left\langle S_{i j}\right\rangle_{F}\left\langle S_{i j}\right\rangle_{H}+(1-k) v_{E}\left\langle S_{i j}\right\rangle_{E}\left\langle S_{i j}\right\rangle_{H}-\frac{k(1-k)}{2} G_{i j}^{a}\left\langle S_{i j}\right\rangle_{H}}{\mathcal{S}^{2}},
$$

where $\mathcal{S}^{2}=\left\langle S_{i j}\right\rangle_{H}\left\langle S_{i j}\right\rangle_{H}$. This procedure has some resemblance to the dynamic eddyviscosity model of Lilly [9]. Analytically, the H-level subgrid scale stress determined using the equivalent eddy-viscosity ((28) and (31)) should be the same as that computed directly using (27). However, direct computation of the H-level subgrid scale stress using (27) led to a numerical instability due to a lack of dissipation, whereas using the equivalent eddy viscosity ((28) and (31)) produced stable solutions with good modeled and resolved Reynolds shear stresses (see Section 4.4). For further discussions, the reader is referred to Rajamani [12].

\subsection{Blending function and reconstruction}

The blending function, $k$, not only delineates the RANS and LES regions, but also plays a major role in the reconstruction of $F$-field from $H$-filed. Traditional blending functions, which perform RANS only near the walls $(k=0)$ and LES away from the walls $(k=1)$, as shown in Fig. 1, imply $\epsilon=0$ in (15). However, reconstruction of LES field from $H$-field becomes difficult with this choice of $\epsilon$. The problem occurs not near the wall where $k=0$, but around the location where $k$ starts to increase from zero. This is because near the wall, where $k=0$, although the recovery factor $\frac{1-k}{k}($ see $(5))$ is undefined, a RANS-only simulation in this region means $\left\langle u_{i}\right\rangle_{H}=\left\langle u_{i}\right\rangle_{E}$. As we reach the cusp of the RANS-LES interface, some LES will be performed and hence the term $\left\langle u_{i}\right\rangle_{H}-\left\langle u_{i}\right\rangle_{E}$ is no longer zero. The recovery factor at this location is, however, large owing to the occurrence of a very small $k$ in the denominator. One way to overcome this problem is to construct a blending function with $0<\epsilon \ll 1$, i.e., $k \neq 0$ near the wall. The implication of a non-zero $\epsilon$ is obvious: some large-scale fluctuations are captured by a coarse LES. We performed all our simulations in this paper with a non-zero $\epsilon$. 
In solving (26) for the $H$-field, we need to reconstruct the $E$ - and $F$-field. Reconstruction of the $E$-field is straightforward from (3). Reconstruction of the $F$ field from the $H$-field, on the other hand, is an ill-posed deconvolution problem and is an inherently noisy process. This is indeed a weakness of the present hybrid-filter approach. In our simulations, $F$-level velocity field was replaced by $H$ - and $E$-level velocity by using (4). The hybrid-filtered Navier-Stokes equations and the continuity equation in terms of $H$ - and $E$-level velocity are then:

$$
\begin{gathered}
\frac{\partial\left\langle u_{i}\right\rangle_{H}}{\partial t}+\frac{\partial\left\langle u_{i}\right\rangle_{H}\left\langle u_{j}\right\rangle_{H}}{\partial x_{j}}+\frac{\widehat{\mathrm{d}\langle P\rangle_{E}}}{\mathrm{~d} x_{1}} \delta_{1 i}+\frac{\widehat{\partial\left\langle P^{\prime}\right\rangle_{H}}}{\partial x_{i}}-\frac{\partial}{\partial x_{j}}\left[\left(\frac{1}{R e}+v_{H}\right) \frac{\partial\left\langle u_{i}\right\rangle_{H}}{\partial x_{j}}\right] \\
=\frac{\partial k}{\partial x_{j}}\left[\left(\left\langle u_{i}\right\rangle_{F}\left\langle u_{j}\right\rangle_{F}-\left\langle u_{i}\right\rangle_{E}\left\langle u_{j}\right\rangle_{E}\right)^{a}-2\left(v_{F}\left\langle S_{i j}\right\rangle_{F}-v_{E}\left\langle S_{i j}\right\rangle_{E}\right)-\frac{2}{R e} \frac{\partial}{\partial x_{j}}\left(\frac{\left\langle u_{i}\right\rangle_{H}-\left\langle u_{i}\right\rangle_{E}}{k}\right)\right] \\
-\frac{1}{R e} \frac{\partial^{2} k}{\partial x_{j}^{2}}\left(\frac{\left\langle u_{i}\right\rangle_{H}-\left\langle u_{i}\right\rangle_{E}}{k}\right)+v_{H} \frac{\partial}{\partial x_{i}}\left[\frac{\partial k}{\partial x_{j}}\left(\frac{\left\langle u_{j}\right\rangle_{H}-\left\langle u_{j}\right\rangle_{E}}{k}\right)\right]+\frac{\partial v_{H}}{\partial x_{j}} \frac{\partial\left\langle u_{j}\right\rangle_{H}}{\partial x_{i}}, \quad \text { (32) } \\
\frac{\partial\left\langle u_{j}\right\rangle_{H}}{\partial x_{j}}=\frac{1}{k} \frac{\partial k}{\partial x_{j}}\left(\left\langle u_{j}\right\rangle_{H}-\left\langle u_{j}\right\rangle_{E}\right) .
\end{gathered}
$$

Similarly, the Germano stresses were obtained from

$$
G_{i j}^{a}=\frac{1-k}{k}\left[\left(\left\langle u_{i}\right\rangle_{H}-\left\langle u_{i}\right\rangle_{E}\right)\left(\left\langle u_{j}\right\rangle_{H}-\left\langle u_{j}\right\rangle_{E}\right)\right]^{a} .
$$

\subsection{Numerical methods}

Equations (32)-(34) were solved using the fractional-step method of Kim and Moin [7]. The viscous terms were treated implicitly using the Crank-Nicolson method, and all other terms were treated explicitly using a low-storage third-order Runge-Kutta method. The Poisson equation for pressure, which was necessary to enforce the continuity equation, in the same manner as enforcing the divergence-free continuity equation for LES or DNS, was solved using a hybrid of fast transformation and a multigrid method (the multigrid method was not necessary for channel flows, but the code was written for generalized coordinates in order to handle more complex geometries). The accuracy of this finite-difference code was validated against the spectral code of Kim et al. [8] for DNS of a regular channel flow. For further details on the numerical methods and validations, the reader is referred to Rajamani [12].

\subsection{Simulation results}

We first performed, as a benchmark case, a simulation for the same Reynolds number, grid and blending function as those used in the a priori tests presented in Section 3. The grid used for the study was $129 \times 129 \times 129$ with $\Delta y_{w}^{+}=0.6$ and $\Delta y_{c}^{+}=2.4$ and the blending function $k$ as given in Fig. $1(\epsilon=0.15)$. The mean velocity profile obtained from the HFNS and that obtained from LES on the same grid are shown together in Fig. 7a, indicating a good agreement. Note that there is no overshoot in the log region and beyond in contrast to those obtained from most existing hybrid approaches (without the special ad hoc forcing). In fact, the $H$-filter 

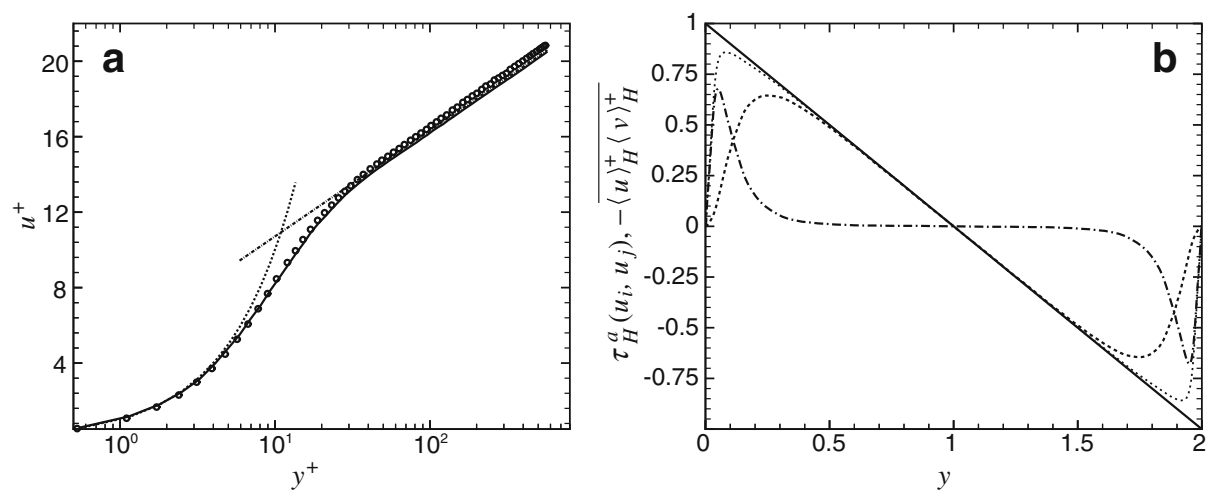

Fig. 7 a Mean velocity profile, $R e_{\tau}=560$ : solid line current $H$-filter result; open circle LES; dashed line $u^{+}=y^{+}$; dash-dot-dashed line $u^{+}=\frac{1}{\kappa} \log y^{+}+5.0$. b Reynolds shear stress: dash-dot-dashed line modeled stress; dashed line resolved stress; dotted line total = modeled + resolved; solid line $1-y$

velocity profile follows the log law (with a logarithmic intercept of $B=5.0$ ) more closely than the LES profile. On this grid, the logarithmic intercept of the LES profile was determined to be 5.1.

For any hybrid method, including DES, an important test for the robustness of the model is the behavior of the Reynolds shear stress at or around the RANS/LES interface. Here, the total stress has to be sustained by both modeled and resolved stresses. Figure $7 \mathrm{~b}$ shows that the modeled and resolved stresses budget themselves at the interface so that the total stress follows the $1-y$ line closely.

Having validated the hybrid filter approach at $R e_{\tau}=560$, we performed several simulations at $R e_{\tau}=5,000$ in order to evaluate its performance at high Reynolds numbers, for which LES would require much finer grids than those described below. We used several different blending functions and two different grids in the wallnormal direction in order to examine the sensitivity. The motivation for using two different wall-normal grids was based on our observation that DES results often suffer from the lack of grid convergence due the fact that RANS/LES region varies as the wall-normal grid varies. We used $129 \times 97 \times 129$ (grid "g1") and $129 \times 129 \times 129$ (grid "g2"), the domain size was $2 \pi \times 2 \times \pi$, respectively, for the streamwise, wallnormal and spanwise directions. Thus, $\Delta x=0.049$ and $\Delta z=0.025$ respectively. For the wall-normal direction, $\Delta y_{w}^{+}=1.43$ and $\Delta y_{c}^{+}=350$ for $129 \times 97 \times 129$ grid and $\Delta y_{w}^{+}=1.2$ and $\Delta y_{c}^{+}=225$ for $129 \times 129 \times 129$ grid. Six cases to be discussed in this paper are shown in Table 1, and different blending functions used are shown in Fig. 8.

Table 1 Flow simulation cases performed

\begin{tabular}{llrlllc}
\hline Case & $R e_{\tau}$ & \multicolumn{1}{c}{$N_{y}$} & $k$ & $\Delta y_{w}^{+}$ & $\Delta y_{c}^{+}$ & $C_{f} \%$ err \\
\hline k00g15K & 5,000 & 97 & Fig. 8a & 1.43 & 350 & 5.56 \\
k01g15K & 5,000 & 97 & Fig. 8a & 1.43 & 350 & 6.24 \\
k02g15K & 5,000 & 97 & Fig. 8a & 1.43 & 350 & 11.32 \\
k03g25K & 5,000 & 129 & Fig. 8b & 1.2 & 225 & 4.19 \\
k04g25K & 5,000 & 129 & Fig. 8b & 1.2 & 225 & 5.01 \\
k05g25K & 5,000 & 129 & Fig. 8b & 1.2 & 225 & 5.19 \\
\hline
\end{tabular}



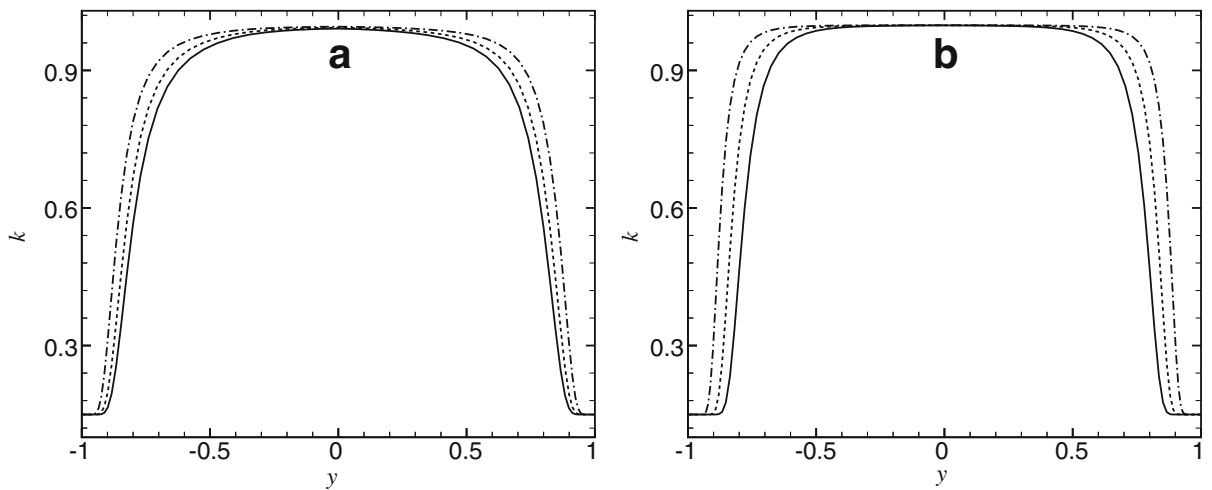

Fig. 8 Different blending functions used in this study generated using different $\alpha$ and $\beta$ (see (15)). a On grid $129 \times 97 \times 129$ ("g1" cases): solid line k00g15K; dashed line k01g15K; dash-dot-dashed line k02g15K. b On grid $129 \times 129 \times 129$ ("g2" cases): solid line k03g25K; dashed line k04g25K; dash-dotdashed line $\mathrm{k} 05 \mathrm{~g} 25 \mathrm{~K}$

Simulations performed on many other grids and blending functions can be found in Rajamani [12].

\subsubsection{Mean velocity profile}

Figure 9 shows mean velocity profiles for six cases. Also plotted in Fig. 9a is a result from DES using the same grid as other cases in the figure. In contrast to the DES result, which shows the typical overshoot (caused in part due to an under-prediction of the skin friction), all $H$-field simulations follow the log law very well. The case $\mathrm{k} 02 \mathrm{~g} 15 \mathrm{~K}$, which had the smallest RANS region, shows a deviation from the log law in the central region. For the combination of the grid and blending function used
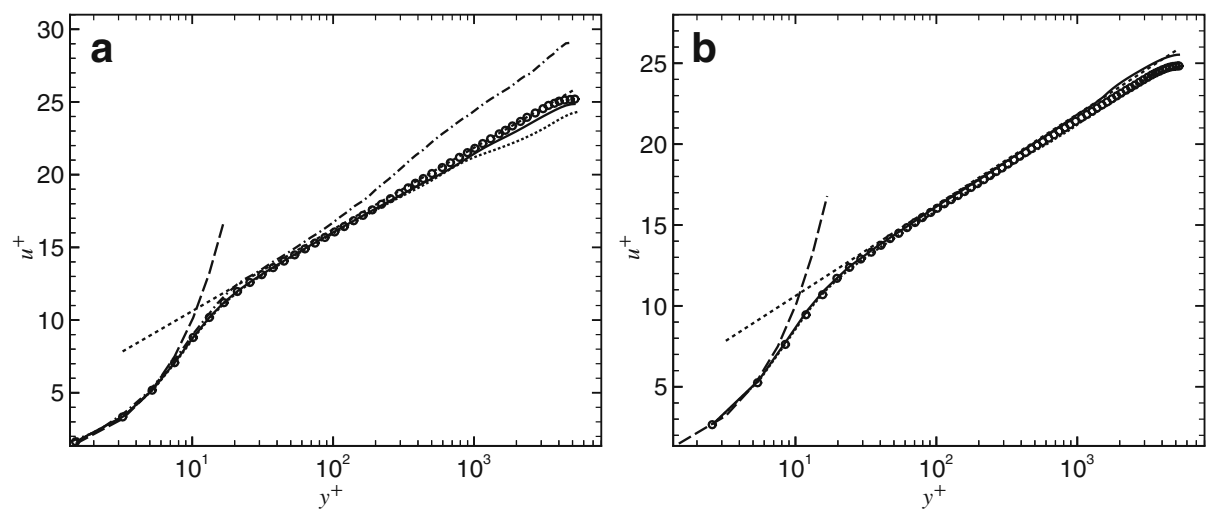

Fig. 9 Mean velocity profiles. a Solid line k00g15K; circle k01g15K; dotted line, k02g15K; dash-dotdashed line $\mathrm{DES}$ on same grid. b solid line $\mathrm{k} 03 \mathrm{~g} 25 \mathrm{~K}$; circle $\mathrm{k} 04 \mathrm{~g} 25 \mathrm{~K}$; dotted line $\mathrm{k} 05 \mathrm{~g} 25 \mathrm{~K}$. In both a and $\mathbf{b}$ : dashed line $u^{+}=y^{+} ;$dotted line $u^{+}=\frac{1}{\kappa} \log y^{+}+5.0$ 
in this case, near-wall region was under-resolved for an early LES. The success of the hybrid-filter approach in reproducing the good mean profiles is attributed to the increased momentum transport near the RANS/LES transition region. The skin-friction coefficient, $C_{f}=\frac{\tau_{w}}{\frac{1}{2} \rho U^{2}}$, is another good indicator of the robustness of the RANS/LES transition zone. The absolute values of percentage errors in the skin-friction coefficient compared with Dean's correlation, $C_{f}=0.073 R e^{-0.25}$, are shown in Table 1 . These errors are better than that of typical hybrid approaches and comparable to that obtained with ad hoc forcing (e.g., Piomelli et al. [11]). From the mean velocity profiles and the $C_{f}$ errors, we notice a close correlation between the shape of the former and the model's ability to predict the latter. We conclude that the same mechanisms that caused the poor prediction of the mean velocity profile is the reason for the poor prediction of the skin friction coefficient as well. This is consistent with the explanations of Baggett [2] and Piomelli et al. [11] that the lack of fluctuations in the RANS region induces artificially long and stable near-wall streaks, which were unable to transfer wall-normal momentum, ultimately leading to a wrong skin friction at the wall.

\subsubsection{Reynolds shear stresses}

Reynolds shear stresses—-modeled, resolved and total—are shown in Figs. 10 and 11. The shapes of the modeled and resolved stresses in these figures reveal that if the blending function is flat in the near wall region and then starts to increase, the modeled stress reaches its peak value and falls monotonically. The resolved stresses adjusts its near wall behavior (Figs. 10b and 11b) such that the total stress (Figs. 10c, and 11c) follows the $1-y$ line in a fully-developed state. The behavior of the modeled and resolved stresses is interesting because, at the interface, the blending function, the RANS and LES eddy viscosity models, and the grid resolution together should produce the right amount of "LES content" without depleting the modeled stresses. This is seen to be true for all cases. One reason for the total stresses to have this correct behavior is due to the Germano stress term as we observed in our a priori tests.

\subsubsection{Instantaneous velocity field}

In Section 3.4 we showed, through a priori tests, that the blending function and the extra terms in HFNS, the Germano stress term in particular, would provide
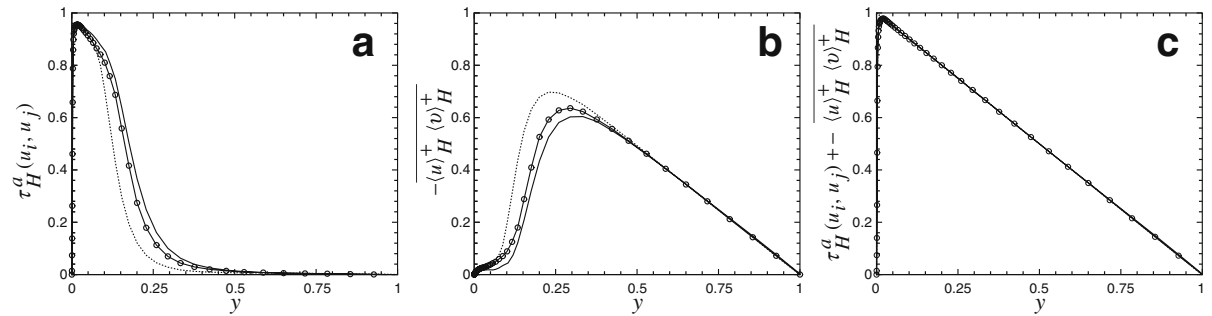

Fig. 10 Reynolds shear stress: a modeled, b resolved $\mathbf{c}$ total. In a, b and $\mathbf{c}$ : solid line case $\mathrm{k} 00 \mathrm{~g} 15 \mathrm{~K}$; dash-open circle-dashed line case $\mathrm{k} 01 \mathrm{~g} 15 \mathrm{~K}$; dotted line case $\mathrm{k} 02 \mathrm{~g} 15 \mathrm{~K}$. In $\mathbf{c}$, dashed line $1-y$ 

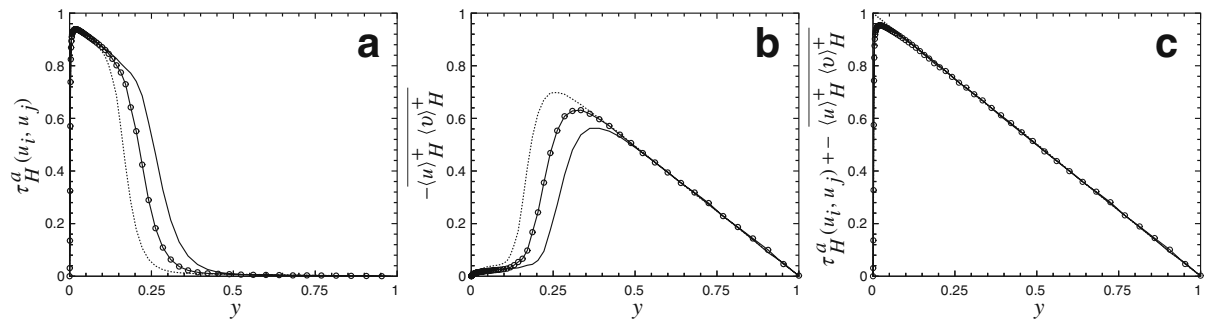

Fig. 11 Reynolds shear stress: a modeled, b resolved $\mathbf{c}$ total. In a, b and c: solid line case $\mathrm{k} 03 \mathrm{~g} 25 \mathrm{~K}$; dash-open circle-dashed line case $\mathrm{k} 04 \mathrm{~g} 25 \mathrm{~K}$; dotted line case $\mathrm{k} 05 \mathrm{~g} 25 \mathrm{~K}$. In $\mathbf{c}$, dashed line $1-y$

additional velocity fluctuations in the RANS/LES transition region. Additional velocity fluctuations would provide adequate momentum transport from RANS to LES region, and would break down the artificially long and stable streaky structures that were observed in many hybrid simulations. This expectation was confirmed in simulations. Consistent with the a priori test results, artificially long streaky structures that extended the entire channel along the streamwise direction were absent (Fig. 12). Such streaks were observed by Baggett [2], Piomelli et al. [11] and in our own DES calculations. In the stochastic forcing case of Piomelli et al. [11], these streaks were seen to be broken down. The present results demonstrate the strength of the hybrid-filter approach in that it does not require any additional ad hoc stochastic forcing in order to have proper momentum transfer in the RANS/LES region, which in turn leads to more accurate turbulence structures in the wall region.
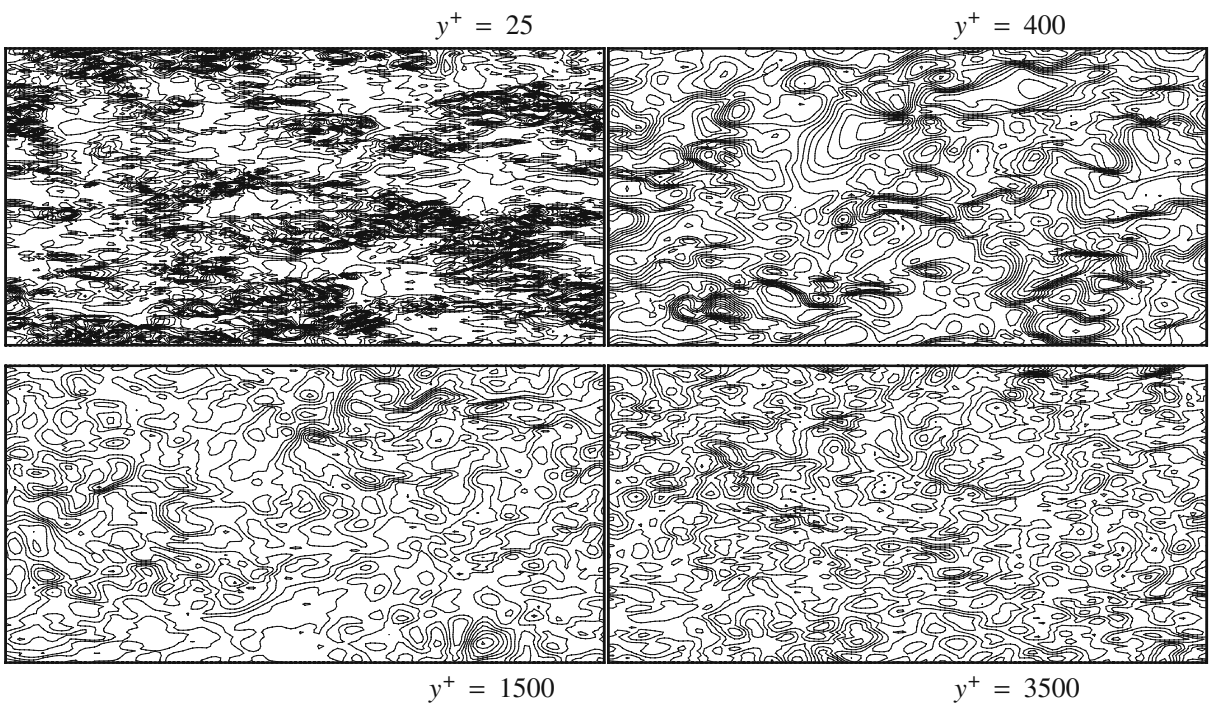

Fig. 12 Contours of streamwise velocity fluctuations, case k02g15K. Plotted for four different wallnormal locations shown 


\section{Summary and Concluding Remarks}

We have examined Germano's hybrid-filter approach, first through a unique a priori test, and then through simulations of turbulent channel flow. We have also addressed the fundamental question of blending two disparate solution techniques. Unlike most existing hybrid methods, which combine two disparate solutions somewhat arbitrarily, Germano's approach defines the combined field in a more precise manner by blending two disparate solutions through a hybrid filter. This process introduces additional terms in the hybrid-filtered Navier-Stokes equations (HFNS). We provided clear evidence that it was essential to have a Germano stress- like term in the Reynolds stresses in order to achieve the correct total stress at the interface. Blending a RANS model and an LES model at a chosen location and matching a secondary quantity such as Reynolds shear stress was not adequate because the Germano stress term (or likewise term) was missing in such approaches (it was brought to our attention by an anonymous referee that Sánchez-Rocha and Menon [13] also used an additive-filter in their hybrid simulation of a compressible boundary layer and drew a similar conclusion). Also clear from the present work was why such methods as the stochastic forcing applied by Piomelli et al. [11] and additional filtering at the interface by Hamba [6] yielded improved results. In these methods, the total Reynolds stresses at the interface was sustained more accurately, albeit artificially, via the additional source terms. It is worth noting that only as an a posteriori exercise, it may be possible to specify the required forcing for a given grid and Reynolds number. It was also shown that the extra terms in HFNS made the transition from the RANS to LES region smoother than a smooth blending function alone can provide, thus also capturing the wall-layer structure correctly.

We were able to successfully simulate the channel flow using the new HFNS equations. The governing equations had several difficulties to overcome. For example, tackling the extra terms in the momentum equation numerically, devising a suitable blending function that minimizes errors in the reconstruction of $F$-field, incorporating a non-solenoidal form of continuity equation into the fractional step method, and finally introducing an equivalent eddy-viscosity to allow enough dissipation to stabilize the $H$-filtered velocity field evaluation were some of the problems we had to overcome in the full simulation of HFNS equations. Despite these difficulties the hybrid-filter approach produced better mean-velocity, skin-friction, Reynolds shear stress and turbulence structures (especially in the RANS/LES region) than other hybrid approaches, including DES, that we have tested. It was shown that the present approach was superior to other hybrid methods in that it did not require any ad hoc forcing in order to have adequate momentum transfer from RANS to LES region.

There are plenty of issues and challenges to overcome before the hybrid-filter approach can be applied to complex turbulent flows at a high Reynolds number, the ultimate goal of all hybrid RANS/LES approaches. Choice of the blending function, appropriate grid resolution (especially in the RANS/LES transition region), and reconstruction of $F$-field from computed $H$-field are among the most critical issues. Based on our observations (some presented in this paper and others not shown here), we draw the following conclusions regarding the blending function and grid resolution:

- The blending function, $k$, can neither be too close to zero in the near-wall region, nor can it be too large. If $k$ is too small, reconstructed LES field is poor; if it is too 
large, higher resolution is required to sustain the increased grid scale stresses. An optimum near-wall value of $k$ appears to be about 0.15 .

- In order for the solution to not be adversely affected in the outer layer, the blending function must have a flat (constant) RANS region. Otherwise, the grid resolution requirements will be excessive in the transition region. This was based on simulations (not shown here) with an extreme blending function, which rises very quickly into the LES region.

- The grid density and blending function are closely related to one another, and the resolution requirement in the near-wall region depends on the shape and value (in the wall region) of the blending function.

- $\quad H$-field solutions did not deteriorate as the grid was refined, in contrast to some DES solutions.

- It is worth noting that $H$-field solutions are different from a numerically underresolved LES field in that all sub-grid scales both at $E$ - and $F$-levels are, at least in principle, properly accounted for by each model. Here we use the term 'filtered' differently from the traditional LES since $H$-filter involves the averaging operator $E$ (equivalent to filtering all fluctuations).

Finally, we conclude that issues and challenges notwithstanding, Germano's hybrid-filter approach appears to be a viable tool for simulating turbulent flows at large Reynolds numbers, for which neither LES nor DNS is feasible in the near future.

Acknowledgements We are grateful to Professor Germano for insightful comments during the course of this work. The numerical simulations were performed at the NCSA Supercomputer center, and the computer times provided through the NSF TRAC program are gratefully acknowledged. On this special occasion of honoring Professor Steve Pope, the second author would like to acknowledge the fruitful interactions and friendship he has enjoyed over the past several decades.

Open Access This article is distributed under the terms of the Creative Commons Attribution Noncommercial License which permits any noncommercial use, distribution, and reproduction in any medium, provided the original author(s) and source are credited.

\section{References}

1. Abe, K.: A hybrid RANS/LES approach using anisotropy resolving algebraic turbulence model. Int. J. Heat Fluid Flow 26(2), 204-222 (2000)

2. Baggett, J.S.: On the feasibility of merging RANS in the near-wall region of attached turbulent flows. Annual Research Briefs, CTR, pp. 267-277. Stanford (1998)

3. Balaras, E., Benocci, C., Piomelli, U.: Finite difference computations of high Reynolds number flows using the dynamic subgrid-scale model. Theor. Comput. Fluid Dyn. 7, 207-216 (1995)

4. Germano, M.: Properties of the hybrid RANS/LES filter. Theor. Comput. Fluid Dyn. 17, 225-331 (2004)

5. Germano, M., Piomelli, U., Moin, P., Cabot, W.H.: A dynamic subgrid scale eddy-viscosity model. Phys. Fluids A 3, 1760-1765 (1991)

6. Hamba, F.: A hybrid RANS/LES simulation of high Reynolds number channel flow using additional filtering at the interface. Theor. Comput. Fluid Dyn. 20(2), 89-101 (2006)

7. Kim, J., Moin, P.: Application of fractional step method to incompressible Navier-Stokes equations. J. Comput. Phys. 59(1), 308-323 (1985)

8. Kim, J., Moin, P., Moser, R.D.: Turbulence statistics in fully developed channel flow at low Reynolds number. J. Fluid Mech. 177, 133-166 (1987)

9. Lilly, D.K.: A proposed modification of the Germano subgrid-scale closure method. Phys. Fluids 4(3), 633-635 (1991) 
10. Nikitin, N.V., Nicoud, F., Wasishto, B., Squires, K.D., Spalart, P.R.: An approach to wall modeling in large eddy simulation. Phys. Fluids 12(7), 1629-1632 (2000)

11. Piomelli, U., Balaras, E., Pasinato, H., Squires, K.D., Spalart, P.R.: The inner-outer layer interface in large-eddy simulations with wall-layer models. Int. J. Heat Fluid Flow 24, 538-550 (2003)

12. Rajamani, B.: Hybrid RANS-LES modeling of High-Re Turbulent Flows. Ph.D. thesis, Department of Mechanical and Aerospace Engineering, UCLA, (2008)

13. Sánchez-Rocha, M., Menon, S.: The compressible hybrid RANS/LES formulation using an additive operator. J. Comput. Phys. 228, 2037-2062 (2009)

14. Shur, M., Spalart, P.R., Strelets, M., Travin, A.: Detached-eddy simulation of an airfoil at high angle of attack. In: Rodi, W., Laurence, D. (eds.) Engineering Turbulence Modeling and Experiments 4. Elsevier, Amsterdam (1999)

15. Spalart, P.R.: Strategies for turbulence modeling and simulations. Int. J. Heat Fluid Flow 21(3), 252-263 (2000)

16. Spalart, P.R., Allmaras, S.R.: A one-equation turbulence model for aerodynamic flows. In: 30th Aerospace Sciences Meeting \& Exhibit, number 92-0439, pp. 1-23. Reno, Nevada (1992)

17. Spalart, P.R., Deck, S., Shur, M.L., Squires, K.D., Strelets, M. Kh., Travin, A.: A new version of detached eddy simulation, resistant to ambiguous grid densities. Theor. Comput. Fluid Dyn. 20(3), 181-195 (2006)

18. Spalart, P.R., Jou, W.-H., Strelets, M., Allmaras, S.R.: Comments on the feasibility of les for wings on a hybrid RANS/LES approach. In: Liu, C., Liu, E. (eds.) Advances in DNS/LES. Greyden, Columbus (1997)

19. Temmerman, L., Hadžabdic̀, M., Leschziner, M.A., Hanjalic̀, K.: A hybrid two-layer URANSLES approach for large eddy simulation at high Reynolds numbers. Int. J. Heat Fluid Flow 26(2), 173-190 (2005)

20. Templeton, J.A., Medic, G., Kalitzin, G.: An eddy-viscosity based near-wall treatment for coarse grid large-eddy simulation. Phys. Fluids A 17, 105101-1-25 (2005)

21. Wang, M., Moin, P.: Dynamic wall modeling for large-eddy simulation of complex turbulent flows. Phys. Fluids 14(7), 2043-2051 (2002) 